\title{
ANÁLISE COMPARATIVA DA INFLUÊNCIA DO TABAGISMO SOBRE A FREQUÊNCIA CARDÍACA A PRESSÃO ARTERIAL E O DUPLO PRODUTO.
}

\author{
Leonardo Squinello Nogueira Veneziano' \\ Criscimar Silva Maia ${ }^{2}$ \\ Nulciene Firmino de Freita ${ }^{3}$ \\ Fernando Duarte Cabra ${ }^{4}$ \\ Rejane Maria Cruvinel Cabral ${ }^{5}$ \\ Roberto Dias ${ }^{6}$ \\ Katia Silveira Ferreira ${ }^{7}$ \\ Lya Carla Manso Miranda ${ }^{8}$ \\ Renata Nascimento Silva ${ }^{9}$ \\ Wilson Alves de Mello ${ }^{10}$ \\ Getulio Antônio de Freitas Filho ${ }^{11}$
}

Resumo: O tabagismo é um dos maiores causadores de doenças cardiovasculares. A nicotina é seu principal componente,causadora de doenças que podem levar à morte, Uma destas doenças é a Hipertensão, que aumenta conforme a alteração no sistema nervoso parassimpático SNP. O objetivo desse estudo é analisar os valores de frequência cardíaca, níveis pressóricos e duplo produto de pacientes tabagistas, não tabagistas e ex-tabagistas. Foi realizada uma pesquisa com 21 exames de holter e MAPA, em um período de um ano, avaliando a frequência cardíaca a pressão arterial e o duplo produto. O estudo analisou todos os valores de frequência cardíaca, pressão arterial sistólica e Duplo-Produto, em exames de holter e MAPA. Os valores de Duplo Produto de fumantes houve aumento de 16,33\%, se comparado aos não-fumantes. Concluindo que existem alterações da frequência cardíaca pressão arterial e Duplo Produto entre pacientes fumantes, não fumantes e ex-fumantes.

Palavras-chave: Pressão arterial; Frequência cardíaca; Duplo produto.

\footnotetext{
${ }^{1}$ Faculdades Objetivo Rio Verde, Brasil. E-mail: leosnv@yahoo.com.br.

2 Faculdades Objetivo Rio Verde, Brasil. E-mail: criscimarmaia13@hotmail.com.

${ }^{3}$ Faculdades Objetivo Rio Verde, Brasil. E-mail: nulciene@faculdadeobjetivo.com.br.

${ }^{4}$ Faculdades Objetivo Rio Verde, Brasil. E-mail: fernandofisio2@hotmail.com.

${ }^{5}$ Faculdades Objetivo Rio Verde, Brasil. E-mail: rejane@faculdadeobjetivo.com.br.

${ }^{6}$ Faculdades Objetivo Rio Verde, Brasil. E-mail: fisiodias@uol.com.br.

7 Faculdades Objetivo Rio Verde, Brasil. E-mail: katiafisio1@bol.com.br.

${ }^{8}$ Faculdades Objetivo Rio Verde, Brasil. E-mail: Iya@faculdadeobjetivo.com.br.

${ }^{9}$ Faculdades Objetivo Rio Verde, Brasil. E-mail: renata@faculdadeobjetivo.com.br.

${ }^{10}$ Faculdades Objetivo Rio Verde, Brasil. E-mail: wilson@faculdadeobjetivo.com.br.

11 Faculdades Objetivo Rio Verde, Brasil. E-mail: getulio@faculdadeobjetivo.com.br.
} 